\title{
Remarks on Yu Miao and Shoufang Xu's paper" Almost sure convergence of weighted sums"
}

João Lita da Silva 


\title{
REMARKS ON YU MIAO AND SHOUFANG XU'S PAPER "ALMOST SURE CONVERGENCE OF WEIGHTED SUMS"
}

\author{
JOÃO LITA DA SILVA
}

Received 26 March, 2014

\begin{abstract}
It is pointed out that a general theorem presented in Yu Miao and Shoufang Xu's paper has a serious gap. In this small note, a counterexample for this theorem is provided exhibiting a triangular array of real numbers and a sequence of independent identically distributed random variables for which its conclusion fails. An reformulation of Yu Miao and Shoufang Xu's general theorem is performed making this statement true.
\end{abstract}

2010 Mathematics Subject Classification: 60F15

Keywords: almost sure convergence, triangular arrays, weighted sums

\section{INTRODUCTION}

In paper [11], the authors announced a general theorem (see Theorem 6 of [11], page 175) which supports important improvements in several well-known results about the almost sure convergence of the weighted sum

$$
\sum_{k=1}^{n} a_{n, k} X_{k}
$$

where $\left\{X_{n}, n \geqslant 1\right\}$ is a sequence of random variables and $\left\{a_{n, k}, 1 \leqslant k \leqslant n, n \geqslant 1\right\}$ is a triangular array of constants.

Over the past years, many authors have studied the almost sure convergence of (1.1) (see, for instance, [2-4, 6, 7, 10,13-18]). In 1995, Li, Rao and Wang proved a result in [10] which seems to say that for almost all choices of weights, the law of the Iterated Logarithm fails, establishing what they call the law of Logarithm. Notwithstanding, a specific example with a concrete array of real numbers and sequence of independent identically distributed random variables for which the law of the Iterated Logarithm fails was not included in the quoted paper. Only one year later, Li and Rao presented the desired example (see [9]) which can be used to claim

This work was partially supported by the Fundação para a Ciência e a Tecnologia (Portuguese Foundation for Science and Technology) through the project PEst-OE/MAT/UI0297/2014 (Centro de Matemática e Aplicações). 
that Corollary 3 of [11] is false. Indeed, a simple adaptation of Li and Rao's example can be made to prove that Theorem 6 of [11] is also false. Contrary to what Miao and $\mathrm{Xu}$ declared in page 176 of [11], we cannot rearrange the random sequence $\left\{a_{n, k} X_{k}, 1 \leqslant k \leqslant n, n \geqslant 1\right\}$ when the order of $\left\{a_{n, k}, 1 \leqslant k \leqslant n, n \geqslant 1\right\}$ does not satisfy $a_{n, 1} \geqslant a_{n, 2} \geqslant \ldots \geqslant a_{n, n}$.

\section{Counterexample}

Consider a sequence $\left\{X_{n}, n \geqslant 1\right\}$ of independent identically distributed random variables each with normal distribution with zero mean and unit variance. Let $\mathbf{H}_{n}$ be a sequence of Hadamard matrices of order $n$ (see [1] or [8]) and suppose the $\infty \times \infty$ matrix $\left(h_{n, k}\right)_{n, k \geqslant 1}$ given by

$$
\left(\begin{array}{cccc}
\mathbf{H}_{1} & \mathbf{O} & \mathbf{O} & \ldots \\
\mathbf{O} & \mathbf{H}_{2} & \mathbf{O} & \ldots \\
\mathbf{O} & \mathbf{O} & \mathbf{H}_{4} & \ldots \\
\vdots & \vdots & \vdots & \ddots
\end{array}\right)
$$

Let $\left\{a_{n, k}, 1 \leqslant k \leqslant n, n \geqslant 1\right\}$ be the triangular array of constants defined by

$$
\begin{aligned}
a_{2 n, k} & =\frac{h_{n, k}}{\log \log \log (n+15) \sqrt{n \log \log (n+2)}}, \\
a_{2 n-1, k} & =\frac{1}{\log \log \log (n+15) \sqrt{n \log \log (n+2)}} .
\end{aligned}
$$

Since $\left|h_{n, k}\right| \leqslant 1$ for each $n, k$ we have

$$
\max _{1 \leqslant k \leqslant n}\left|a_{n, k}\right| \leqslant \frac{1}{\log \log \log (n+15) \sqrt{n \log \log (n+2)}}
$$

and the sequence $b_{n}$ in Theorem 6 of Yu Miao and Shoufang Xu's paper is

$$
b_{n}=\log \log \log (n+15) \sqrt{n \log \log (n+2)} .
$$

From Hartman-Wintner law of the iterated logarithm (see [12], page 248) we get

$$
\frac{X_{1}+\ldots+X_{n}}{b_{n}} \stackrel{\text { a.s. }}{\longrightarrow} 0 .
$$

On the other hand,

$$
\limsup _{n \rightarrow \infty}\left|\sum_{k=1}^{n} a_{n, k} X_{k}\right| \geqslant \limsup _{n \rightarrow \infty} \frac{1}{b_{n}}\left|\sum_{k=1}^{2 n} h_{n, k} X_{k}\right| \text { a.s. }
$$

and

$$
\limsup _{n \rightarrow \infty} \frac{1}{\sqrt{4 n \log (2 n)}}\left|\sum_{k=1}^{2 n} h_{n, k} X_{k}\right| \geqslant \frac{1}{\sqrt{2}} \quad \text { a.s. }
$$


(see [9]) which implies

$$
\limsup _{n \rightarrow \infty}\left|\sum_{k=1}^{n} a_{n, k} X_{k}\right|=\infty \text { a.s. }
$$

Therefore, subsequent corollaries in [11] which use Theorem 6 cannot be validated until a correct proof be given.

\section{GenERAL THEOREM}

We shall announce the following definition.

Definition 1. Let $\left\{a_{n, k}, 1 \leqslant k \leqslant n, n \geqslant 1\right\}$ be an array of constants. We say that $\left\{a_{n, k}, 1 \leqslant k \leqslant n, n \geqslant 1\right\}$ is rowwise increasing (resp. rowwise decreasing) if $a_{n, k+1} \geqslant a_{n, k}$, for all $1 \leqslant k \leqslant n-1, n \geqslant 2$ (resp. $a_{n, k+1} \leqslant a_{n, k}$, for all $1 \leqslant k \leqslant n-1$, $n \geqslant 2)$. An array of constants $\left\{a_{n, k}, 1 \leqslant k \leqslant n, n \geqslant 1\right\}$ is said to be rowwise monotone if it is rowwise increasing or rowwise decreasing.

The result below will permit us to obtain the desired almost sure convergence of (1.1) in all corollaries of [11] assuming additionally the rowwise monotonicity of the triangular array of constants. The proof uses Abel transformation (see [5], page 114) and is similar to Cèsaro lemma (see [19], page 116) being, therefore, omitted.

Theorem 1. Let $\left\{x_{n}\right\}$ be a sequence of constants and $\left\{a_{n, k}, 1 \leqslant k \leqslant n, n \geqslant 1\right\}$ an rowwise monotone array of constants satisfying

$$
\max _{1 \leqslant k \leqslant n}\left|a_{n, k}\right| \leqslant b_{n}^{-1}
$$

where $0<b_{n} \uparrow \infty$. If

$$
\frac{1}{b_{n}} \sum_{k=1}^{n} x_{k} \rightarrow 0
$$

then

$$
\sum_{k=1}^{n} a_{n, k} x_{k} \rightarrow 0 .
$$

Remark 1. Let us observe that the assumption " $\left\{X, X_{n}, n \geqslant 1\right\}$ be a sequence of identically distributed random variables" in Theorem 6 of [11] is totally superfluous.

\section{REFERENCES}

[1] S. Agaian, Hadamard Matrices and Their Applications, ser. Lectures Notes in Mathematics. Berlin-Heidelberg: Springer-Verlag, 1985, vol. 1168.

[2] B. Choi and S. Sung, "Almost sure convergence theorems of weighted sums of random variables," Stoch. Anal. Appl., vol. 5, no. 4, pp. 365-377, 1987.

[3] Y. Chow, "Some convergence theorems for independent random variables," Ann. Math. Stat., vol. 37, no. 6, pp. 1482-1493, 1966. 
[4] Y. Chow and T. Lai, "Limiting behavior of weighted sums of independent random variables," Ann. Probab., vol. 1, no. 5, pp. 810-824, 1973.

[5] Y. Chow and H. Teicher, Probability Theory: Independence, Interchangeability, Martingales, 3rd ed., ser. Springer texts in Statistics. New York: Springer, 1997.

[6] J. Cuzick, "A strong law for weighted sums of i.i.d. random variables," J. Theoret. Probab., vol. 8, no. 3, pp. 625-641, 1995.

[7] C. Heyde, "An iterated logarithm result for martingales and its application in estimation theory for autoregressive processes," J. Appl. Probab., vol. 10, pp. 146-157, 1973.

[8] K. Horadam, Hadamard Matrices and Their Applications. New Jersey: Princeton University Press, 2007.

[9] D. Li and B. Rao, "A note on the law of the iterated logarithm for weighted sums of independent identically distributed random variables," in Convergence in Ergodic Theory and Probability, ser. Conference on Convergence in Ergodic Theory and Probability, V. Bergelson, P. March, and J. Rosenblatt, Eds. Berlin: Walter de Gruyter \& Co., 1996, pp. 273-276.

[10] D. Li, M. Rao, and X. Wang, "On the strong law of large numbers and the law of the logarithm for weighted sums of independent random variables with multidimensional indices," J. Multivariate Anal., vol. 52, pp. 181-198, 1995.

[11] Y. Miao and S. Xu, "Almost sure convergence of weighted sums," Miskolc Math. Notes, vol. 14, no. 1, pp. 173-181, 2013.

[12] V. Petrov, Limit Theorems of Probability Theory: Sequences of Independent Random Variables, ser. Oxford Studies in Probability. New York: Oxford University Press, 1995, vol. 4.

[13] Q.-M. Shao, "Maximal inequalities for partial sums of $\rho$-mixing sequences," Ann. Probab., vol. 23, no. 2, pp. 948-965, 1995.

[14] Q.-M. Shao and C. Su, "The law of the iterated logarithm for negatively associated random variables," Stochastic Process. Appl., vol. 83, pp. 139-148, 1999.

[15] S. Sung, "Almost sure convergence for weighted sums of i.i.d. random variables (II)," Bull. Korean Math. Soc., vol. 33, no. 3, pp. 419-426, 1996.

[16] S. Sung, "Almost sure convergence for weighted sums of i.i.d. random variables," J. Korean Math. Soc., vol. 34, no. 1, pp. 57-67, 1997.

[17] H. Teicher, "Almost certain convergence in double arrays," Z. Wahrscheinlichkeitstheorie verw. Gebiete, vol. 69, pp. 331-345, 1985.

[18] R. Thrum, "A remark on almost sure convergence of weighted sums," Probab. Theory Related Fields, vol. 75, pp. 425-430, 1987.

[19] D. Williams, Probability with Martingales, ser. Cambridge Mathematical Textbooks. Cambridge University Press, 1991.

Author's address

João Lita da Silva

New University of Lisbon, Faculty of Sciences and Technology, Department of Mathematics and CMA, Quinta da Torre, Caparica, 2829-516, Lisbon, Portugal

E-mail address: jflsefct.unl.pt 